\title{
Parametrizing Drug Effects with Machine Learning in a New Resonant Model of Cell Electrophysiology
}

\author{
Sucheta Sehgal ${ }^{1}$, Nitish D Patel ${ }^{1}$, Mark L Trew ${ }^{2}$ \\ ${ }^{1}$ The University of Auckland, Auckland, New Zealand \\ ${ }^{2}$ Auckland Bioengineering Institute, The University of Auckland, Auckland, New Zealand
}

\begin{abstract}
Drugs often alter the action potential morphologies of cardiac cells. These changes can induce life-threatening arrhythmias. Although models of cellular electrophysiology $(E P)$ are widely available, we have developed a promising alternative: a novel resonant model $(R M)$ designed around the computational strengths of non-CPU platforms such as GPU and FPGA. However, like most models, the RM requires parameterization. To effectively parameterize the RM to capture drug effects on the action potential, we have investigated a machine learning algorithm using a Feed-Forward Neural Network (FFNN). We first designed the FFNN architecture and then found its hyperparameters using a random space search algorithm. The FFNN was trained on data obtained by blocking the 'funny current' in a detailed EP model of the human sinoatrial node (SAN). We used unseen current blockage data as an input to the FFNN for validation. The correlation coefficients between all predicted and reference AP waveform morphologies was above 0.97, with zero and close to zero p-values, indicating significant predictions. Parameters of the RM obtained from FFNN can be used to capture or predict changes in AP morphologies due to drug effects.
\end{abstract}

\section{Introduction}

The mechanisms of drug interaction with the EP of various organs are very complex and the effects span from the ion channel to the whole organ level. This makes an investigation of the drugs using solely experimental techniques very difficult and leads to incompatibility of prescription drugs with precision health. Computational models have provided insights into the mechanisms of the lifethreatening phenomena, such as cardiac arrhythmogenesis [1]. Therefore, these models represent a powerful tool in investigating drug effects [2]. Also, promotion of computational models to quantify the effects of drugs has been recommended by CiPA [3]. Recently, we have developed a new high-fidelity computational model of AP of biolog- ical cells [4]. In this paper, we propose a neural network model that can predict the parameters of the RM that correspond to a specific value of a current blockage. We show the efficacy of the proposed RM parameterization to predict changes in AP morphologies and we quantify the validity of the predicted AP waveforms by correlating them to the reference AP waveforms. Also, the comparison has been made between the FFNN fits and other parameterization methods (piecewise linear fits (PLF) and mixed fits (MF)) developed in our previous work [4].

\section{Methods}

Our objective is to develop an artificial neural network that predicts the RM coefficients with alterations in the ionic channel due to the effects of drugs. In this section, we describe the RM, general neural network architecture and the selection of the hyperparameters of the proposed architecture.

Resonant model of a biological cell (as detailed in [4]) uses finite trigonometric series in Equation (1) to generate the electrical pulse output of a cell $V(t, \beta)$.

$$
V(t, \beta)=a_{0}(\beta)+\sum_{i=1}^{n} c_{i}(\beta) \cos \left(i \omega(\beta) t-\phi_{i}(\beta)\right)
$$

Here, $\beta$ represents the percentage reduction in an ion channel conductivity by the administration of a drug. The dynamic parameters $\left(a_{0}, c_{i}, \omega, \phi_{i}\right)$ of the RM capture the change in the electrical pulse output due to the influence of a drug. As shown in Figure 1A, the FFNN generates these parameters and feed to the waveshape generator (WG). A WG consisting of $n$ oscillators then produces an action potential (AP) waveshape corresponding to a value of $\beta$.

The reference data to train and test the FFNN was obtained using the Fabbri et al. model of human SAN [5]. The model was simulated by varying the maximum funny current $\left(I_{f}\right)$ conductance $g_{f}$ with $\beta$ lying between 0 and 1 such that $g_{f}^{\prime}=\beta * g_{f}$. For training, eleven different AP waveshapes were obtained corresponding to eleven equally spaced values of $\beta$. The coef- 

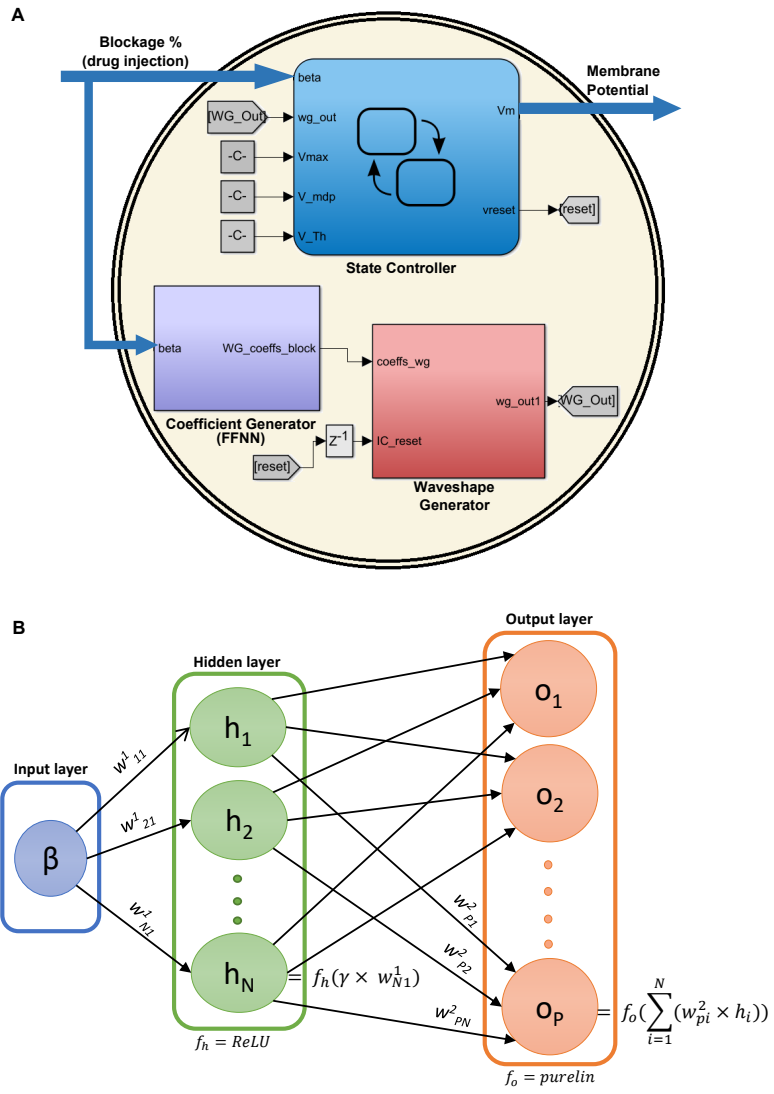

Figure 1. A: Schematic diagram of the Resonant model of a biological cell parameterized with the feed-forward neural network (FFNN). B: The layout of the FFNN architecture.

ficients of the RM (with $n$ equals ten i.e. 22 parameters $\left.\left(a_{0} ; a_{1}, . ., a_{10} ; b_{1}, . ., b_{10} ; \omega\right)\right)$ for each of the eleven APs were obtained using the methodology presented in [4]. The $\beta$ values lying between the training values were used for obtaining the AP waveshapes for validating the FFNN. As all the parameters of the RM (output data of FFNN) have different ranges, they are all normalized before training to improve the convergence speed of the algorithm. The normalized data $D_{i}^{N}$ was obtained in the range 0-1 using, $D_{i}^{N}=\frac{D_{i}-D_{\min }}{D_{\max }-D_{\min }}$, where $D_{\max }$ and $D_{\min }$ are the extreme values of the parameter $D_{i}$.

The FFNN architecture that forms the basis for predicting the RM parameters is shown in Figure 1B. The FFNN consists of: (1) an input layer to read the input signal. In this study, the input layer has one input i.e. $\beta$. (2) A hidden layer, consists of $N$ neurons, performs computation on the weighted $\beta$ to discover the relationship between the $\beta$ and the RM parameters. (3) An output layer which consists of $P$ neurons to produce $P$ number of parameters of the RM. Each neuron connects to the neurons of the adjacent layer. In the present work, RM has 22 parameters. The $\omega$ in Equation (1) was fitted with piecewise linear equations as in [4]. Other $21 \mathrm{RM}$ parameters were obtained from the output layer of the FFNN. The strength of the connection is indicated by the weight, e.g. $w_{N 1}^{1}$, associated with it. The output of each neuron is obtained from the activation function fed with the sum of weighted inputs. To achieve the best computational performance a rectified linear unit activation function $\left(f_{h}\right)$ was used in the hidden layer and linear function $\left(f_{o}\right)$ was considered for the neurons in the output layer.

Training the FFNN involves the tuning of the hyperparameters for the FFNN to show efficacy. Table 1 shows the values of the hyperparameters searched through during the tuning process using TensorFlow. It was found that the best results for the majority of the RM parameters of amplitude one or more than one were obtained using FFNN with 2 neurons in the hidden layer with 'AdamOptimizer' as the optimization algorithm and 0.005 as the learning rate. The RM parameters of amplitude close to one were predicted better with 3 neurons in the hidden layer. However, the difference in the performance was small enough to choose less computationally expensive FFNN of 2 hidden layer neurons for the parametrization of the RM. These hyperparameters were then used to find the weight vector of each neuron in the network that minimizes the sum of the root mean square errors between the predicted and the original 21 RM parameters.

Table 1. Values explored in the hyperparameter tuning process.

\begin{tabular}{lr}
\hline \hline Hyperparameter & Values explored \\
\hline Learning rate & $0.001,0.005,0.01,0.05$ \\
Hidden layer neurons & $1,2,3,4,5,10$ \\
Optimization algorithm & RMSProp, \\
& GradientDescent, Adam \\
\hline \hline
\end{tabular}

\section{Results and Discussion}

We present the results for the developed FFNN which makes the RM capable of producing the electrophysiological effects of drugs. Figure 2 shows the variations in the $\mathrm{RM}$ parameters as a function of $\beta: a_{1}(\beta), b_{1}(\beta)$ and so on, and the FFNN fits to these parameters by which an AP waveshape can be constructed at an arbitrary $\beta$ between 0 and 1 . The percentage RMSE for twenty interpolated values of $\beta$ is shown using light blue bars in Figure 3. This error measures the deviation of the AP waveform generated by the RM when the coefficients were determined from the FFNN and when the coefficients were obtained after fitting to Fabbri et al. model's reference AP waveform. In this figure, the comparison was made when the 

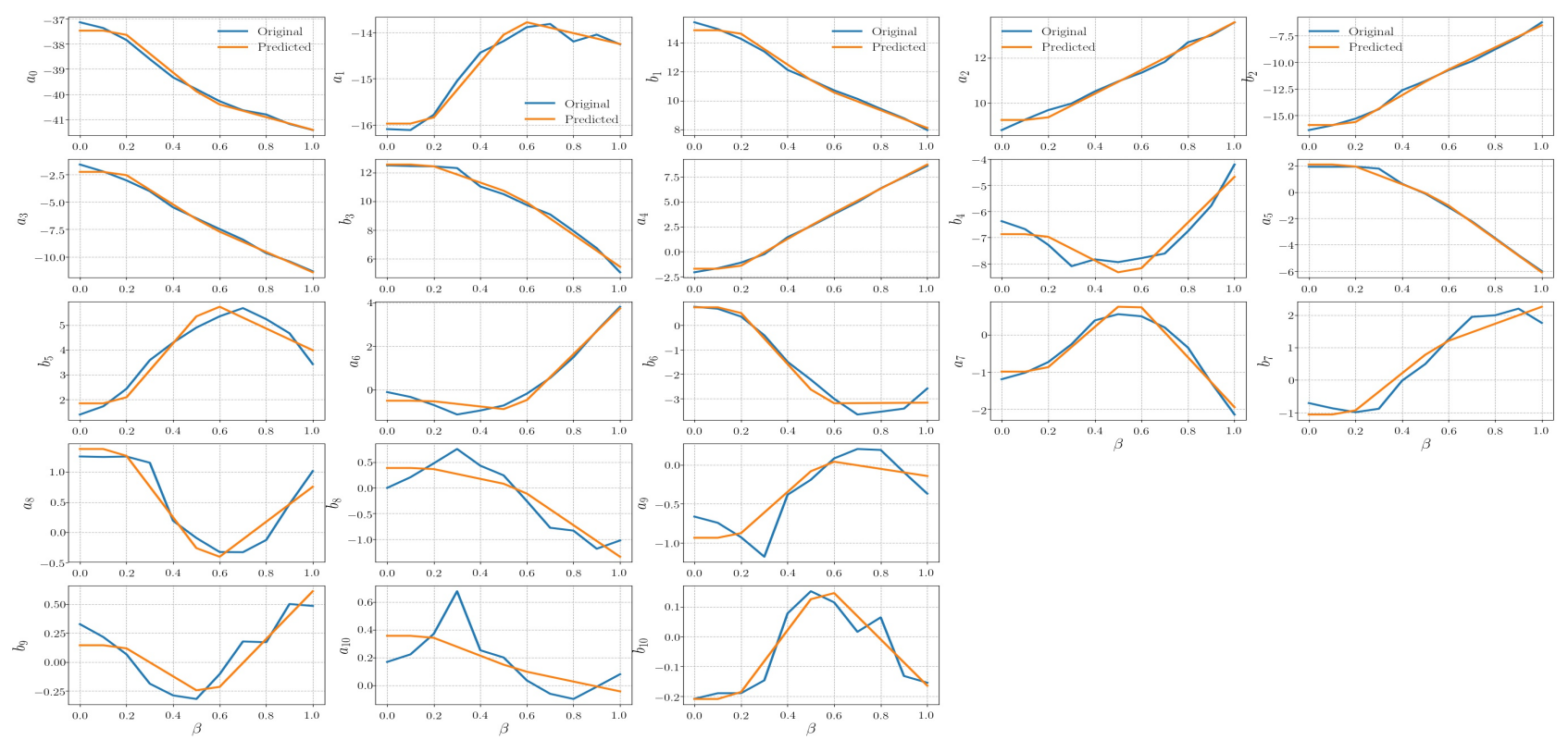

Figure 2. Profiles of the Resonant model parameters with changing 'funny current'. The blue traces are the training data obtained by parameterizing the detailed model waveshapes. The orange traces are the predictions of FFNN with 2 neurons.

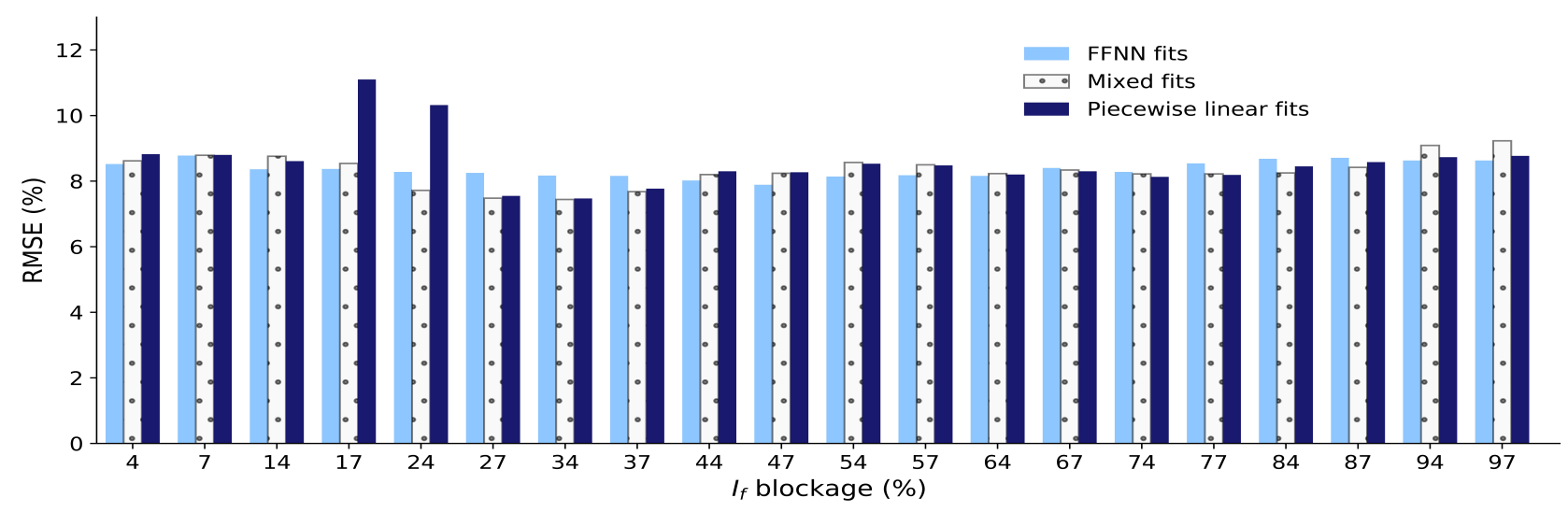

Figure 3. Percentage root mean squared error (RMSE) comparison of different Resonant model parameterization techniques for $20 I_{f}$ blockage percentage values. The coefficients were fitted with: 1) feed-forward neural network (light blue), 2) minimum RMSE fits (white dotted), and 3) minimum piecewise linear fits (dark blue).

RM was parameterized with PLF (dark blue bars) and MF (white dotted bars) as detailed in our previous work [4]. The figure shows that for most of the funny current blockage levels, the FFNN predictions outperform PLF and MF. At some blockage ranges (e.g 27\%-37\%), MF performs slightly better.

Figure 4 shows the predicted AP waveforms by the FFNN and PLF parameterized RM, $\mathrm{RM}_{\mathrm{NN}}$ and $\mathrm{RM}_{\mathrm{PL}}$, respectively, for the four different $I_{f}$ blockage values. The waveforms are compared to the corresponding $I_{f}$ blockage simulations using the detailed (Fabbri et al.) EP model of the human SAN. Note here that the correlation coefficients $\mathrm{CC}_{\mathrm{NN}}$ (between detailed model's and FFNN parameterized
RM's AP waveforms) and $\mathrm{CC}_{\mathrm{PL}}$ (between detailed model's and PLF parameterized RM's AP waveforms) for all the predicted AP waveforms are above 0.97 and approaching the ideal value of 1 . All the predicted AP waveforms and those obtained from Fabbri et al. model are significantly correlated $(p=0)$. Figure 5 draws out a comparison of predicted AP waveform CL metric and shows that the CL closely tracks the Fabbri et al. model. The quantitative effect of the total block of $I_{f}$ in the FFNN parameterized RM led to an increase in CL of $27.6 \%$. This was in good accordance with the $26 \%$ increase in the CL that was experimentally observed by Verkerk et al. [6] upon the complete 
Table 2. Cost comparison of the RM parameterization methods.

\begin{tabular}{lccc}
\hline \hline & PLF $^{1}$ & MF $^{2}$ & FFNNF $^{1}$ \\
\hline Coefficients & 170 & 189 & 96 \\
Additions & 22 & 143 & 65 \\
Multiplications & 22 & 644 & 45 \\
Conditionals & 85 & 10 & 6 \\
\hline \hline
\end{tabular}

${ }^{1} \overline{\text { Piecewise linear fits, }{ }^{2} \text { Mixed fits, }{ }^{3} \text { Feed-forward neural network fits }}$

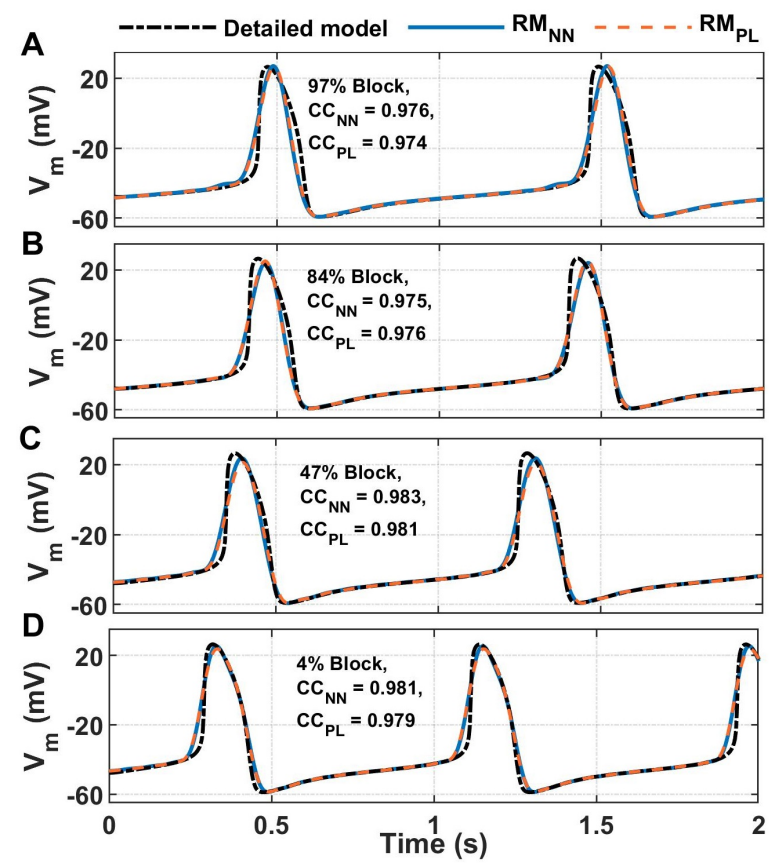

Figure 4. AP waveforms simulated in human SAN cell detailed EP model and parameterized Resonant model (prediction results) upon A: 97\%, B: $84 \%, \mathrm{C}: 47 \%$, and D: $4 \%$ block of $I_{f}$. The correlation coefficients $\left(\mathrm{CC}_{\mathrm{NN}}\right.$ and $\mathrm{CC}_{\mathrm{PL}}$ ) between the reference and parameterized RM AP waveforms are also shown.

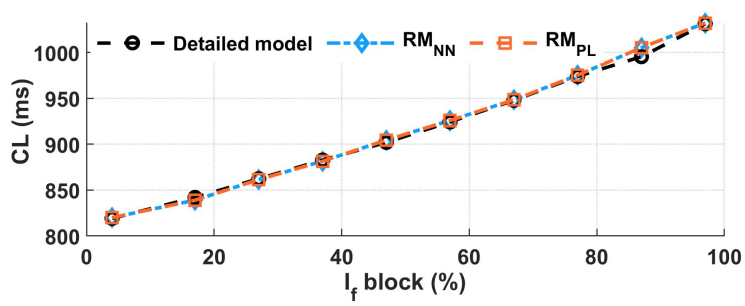

Figure 5. Predicted effects of $I_{f}$ blockage on cycle length (CL).

blockage of $I_{f}$ by the administration of a $2 \mathrm{mM} \mathrm{Cs}^{+}$.
Table 2 shows the number of floating-point operations required to generate the RM coefficients with the different fit types and the number of coefficients to be stored in the memory. Even after accounting the capabilities of various implementation platforms such as CPU, FPGA, and GPU, the computational cost of MF far exceeds the accuracy benefits.

\section{Conclusion}

A machine learning algorithm is effective for parameterizing a new resonant model of cell electrical behaviour, enabling the capture of current blockage effects. With these parameterizations, the computational strengths of RM models can be applied to study and predict the effects of drugs on large scale simulations of cardiac electrical activity. In future, we hope to develop more complex neural network models to capture multiple ion channel blockage effects.

\section{References}

[1] Sahli Costabal F, Yao J, Kuhl E. Predicting drug-induced arrhythmias by multiscale modeling. International Journal for Numerical Methods in Biomedical Engineering 2018; 34:e2964.

[2] Mirams GR, Cui Y, Sher A, Fink M, Cooper J, Heath BM, McMahon NC, Gavaghan DJ, Noble D. Simulation of multiple ion channel block provides improved early prediction of compounds' clinical torsadogenic risk. Cardiovascular Research 2011;91:53-61.

[3] Colatsky T, Fermini B, Gintant G, Pierson JB, Sager P, Sekino Y, Strauss DG, Stockbridge N. The comprehensive in vitro proarrhythmia assay (cipa) initiative - update on progress. Journal of Pharmacological and Toxicological Methods 2016;81:15 - 20.

[4] Sehgal S, Patel ND, Malik A, Roop PS, Trew ML. Resonant model-a new paradigm for modeling an action potential of biological cells. PLOS ONE 2019;14:1-25.

[5] Fabbri A, Fantini M, Wilders R, Severi S. Computational analysis of the human sinus node action potential: model development and effects of mutations. The Journal of physiology 2017;595:2365-2396.

[6] Verkerk AO, Wilders R, van Borren MM, Peters RJ, Broekhuis E, Lam K, Coronel R, de Bakker JM, Tan HL. Pacemaker current (If) in the human sinoatrial node. European Heart Journal 2007;28:2472-2478.

Address for correspondence:

Nitish D. Patel

20 Symonds Street, Faculty of Engineering, Building 401

The University of Auckland, Auckland 1010, New Zealand nd.patel@auckland.ac.nz 